\title{
Smart Watch Detection of Supraventricular Tachycardia (SVT): First Case from Tanzania
}

\author{
Nadeem Kassam (iD) \\ Omar Aziz' \\ Eric Aghan ${ }^{2}$ \\ Samina Somji ${ }^{1}$ \\ Hanifa Mbithe' \\ Mustafa Bapumia ${ }^{3}$ \\ Robert Mvungi ${ }^{3}$ \\ Salim Surani ${ }^{4}$
}

'Department of Internal Medicine, Aga Khan University Medical College East Africa (Dar), Dar es Salaam, Tanzania; ${ }^{2}$ Department of Family Medicine, Aga Khan University Medical College East Africa (Dar), Dar es Salaam, Tanzania; ${ }^{3}$ Department of Internal Medicine and Cardiology, Aga Khan Hospital, Dar es Salaam, Tanzania; ${ }^{4}$ Department of Medicine \& Pharmacy, Texas A \& M University, College Station, TX, USA
Correspondence: Nadeem Kassam $\mathrm{Tel}+255762903008$

Email Nadeem.kassam@akhst.org

\begin{abstract}
Smartwatches like the Apple Watch have been on the rise worldwide and their use is gaining popularity in developing countries. Their ability to detect dysrhythmias is well documented. Present practice discourages the use of these devices as a diagnostic tool. Nevertheless, atypical findings from these devices should be clinically investigated. This case demonstrates an eventual diagnosis of supraventricular tachycardia (SVT) based on an Apple watch alert which was subsequently confirmed by electrophysiological evaluation.

Keywords: supraventricular tachycardia, Apple watch, Tanzania, electrophysiology study
\end{abstract}

\section{Introduction}

Global sales of wearable smartwatches have grown at an annual rate beyond $20 \%$, with approximately half a billion active users in 2020 which is expected to double by $2022 .{ }^{1}$ The incorporation of photoplethysmographic (PPG) sensors into smartwatches has enhanced extensive community-based heart rate monitoring. The latest version of smartwatches incorporate the PPG sensor that can identify pulse rate and regularity offering precise results directly to the user. Despite having a paucity of scientific evidence to support the use of these devices to detect either symptomatic or malignant arrhythmias. There are numerous anecdotal reports of patients saved by their smartwatches which were able to detect and warn the user of an arrhythmia. ${ }^{1}$ We hereby present a case of a 42-year-old African male who was alerted to a possible arrhythmia by his smart watch and was subsequently diagnosed to have supraventricular tachycardia (SVT).

\section{Case Report}

A 42-year-old male with well-controlled hypertension on once-daily Losartan $50 \mathrm{mg}$ presented with acute chest discomfort associated with palpitations and lightheadedness. He was at home watching television when these symptoms started acutely and continued for almost 10 minutes. In addition, his Apple Watch (Apple Watch Series 4) recorded a high resting heart rate of 210 beats/min (Figure 1). Alarmed at the elevated heart rate, he hurried to the emergency department. On examination, the patient was conscious, awake and oriented with warm peripheral extremities. Initial vitals at the emergency department revealed tachycardia of 184 beats/minute, blood pressure of $120 / 80 \mathrm{mmHg}$ and respiratory rate of 16 cycles per minute; his oxygen saturation was $99 \%$ on room air. Systemic examination was unremarkable. A 12 lead electrocardiogram (ECG) was performed (Figure 2) and revealed supraventricular tachycardia at the rate of $184 \mathrm{~b} / \mathrm{min}$. The patient was 


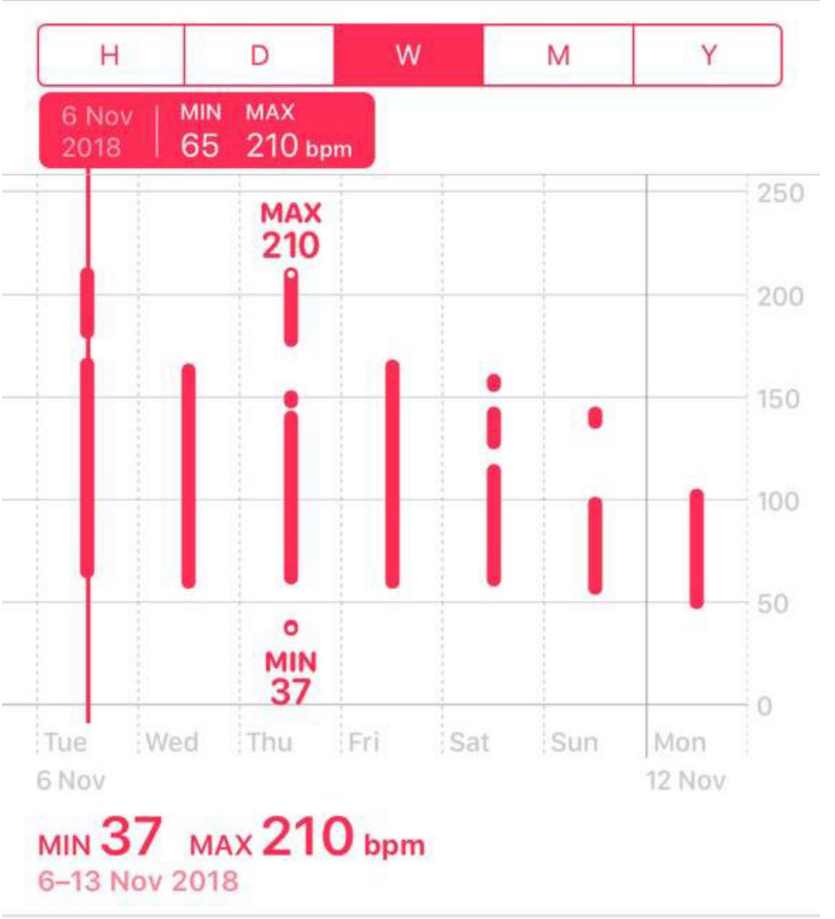

Figure I Apple watch record of the patient's heart rate.

given intravenous Adenosine $6 \mathrm{mg}$ and then two additional doses of $12 \mathrm{mg}$ via cubital peripheral line but failed to revert to a normal sinus rhythm. At this point, he developed hypotension and was quickly electrically cardioverted (synchronized DC 50 Joules) and successfully reverted to normal sinus rhythm as seen in the repeat ECG below (Figure 3). Subsequent workup with a complete blood count, thyroid panel, repeated cardiac enzymes, Troponin I, electrolytes, renal functions were all normal. 2D echocardiogram revealed normal left ventricular systolic (ejection fraction 60\%) and diastolic functions with normal-sized chambers. Coronary angiography demonstrated no evidence of epicardial coronary artery disease. The patient was discharged on daily Nebivolol $5 \mathrm{mg}$, Atorvastatin $20 \mathrm{mg}$ and continued his daily Losartan $50 \mathrm{mg}$ with a plan of electrophysiological studies (EPS) to establish the possible cause of the symptomatic supraventricular tachycardia (SVT). With no EPS studies available in our country, he subsequently flew to India after four weeks and underwent an electrophysiology evaluation which revealed inducible tachycardia on pacing protocols. In addition, electrophysiological mapping showed reproducible SVT, which was orthodromic atrioventricular reentrant tachycardia (oAVRT) utilizing parahisian accessory pathway as retrograde conduction. A ten second ablation was done on the non-coronary

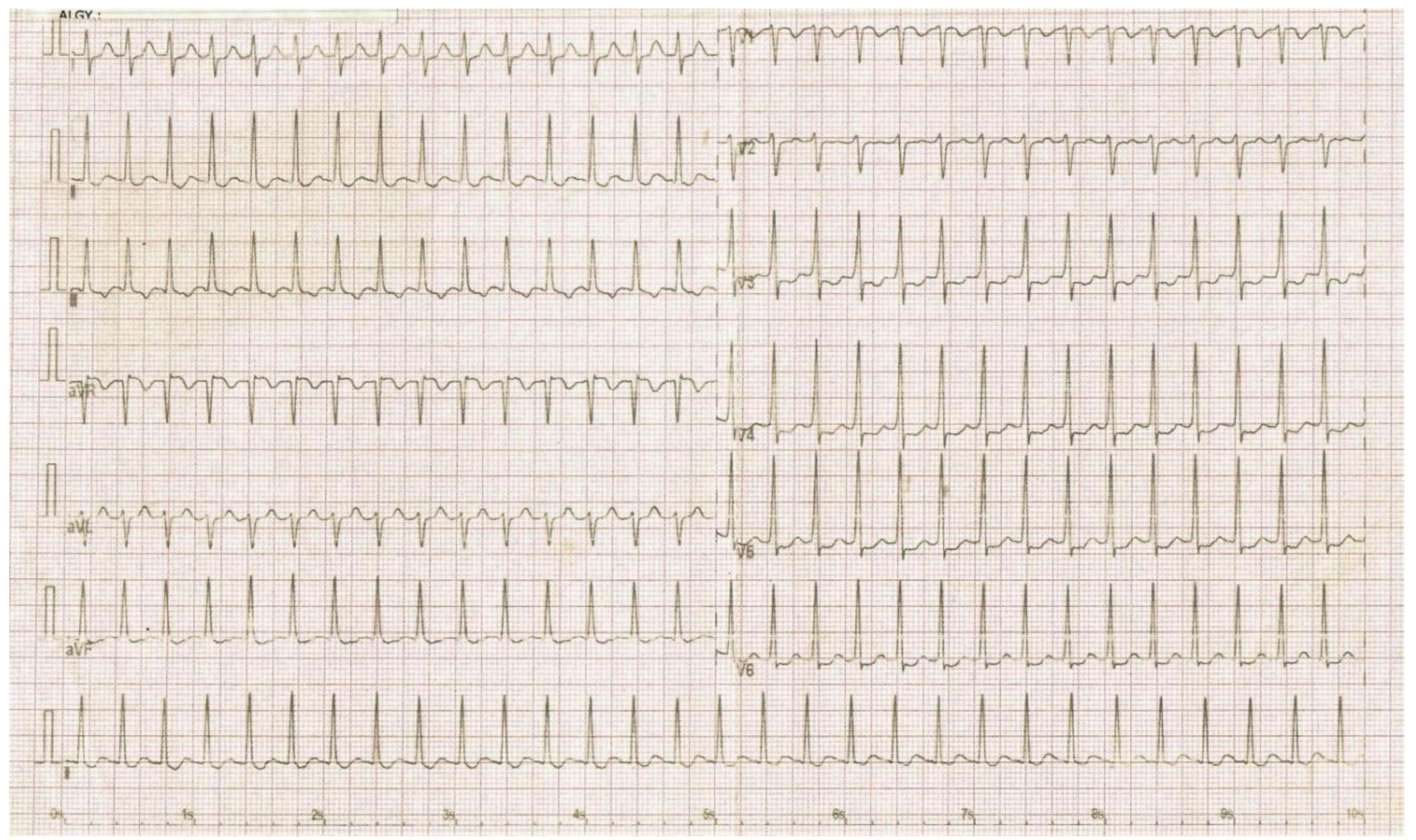

Figure 2 EKG showing supraventricular tachycardia. 


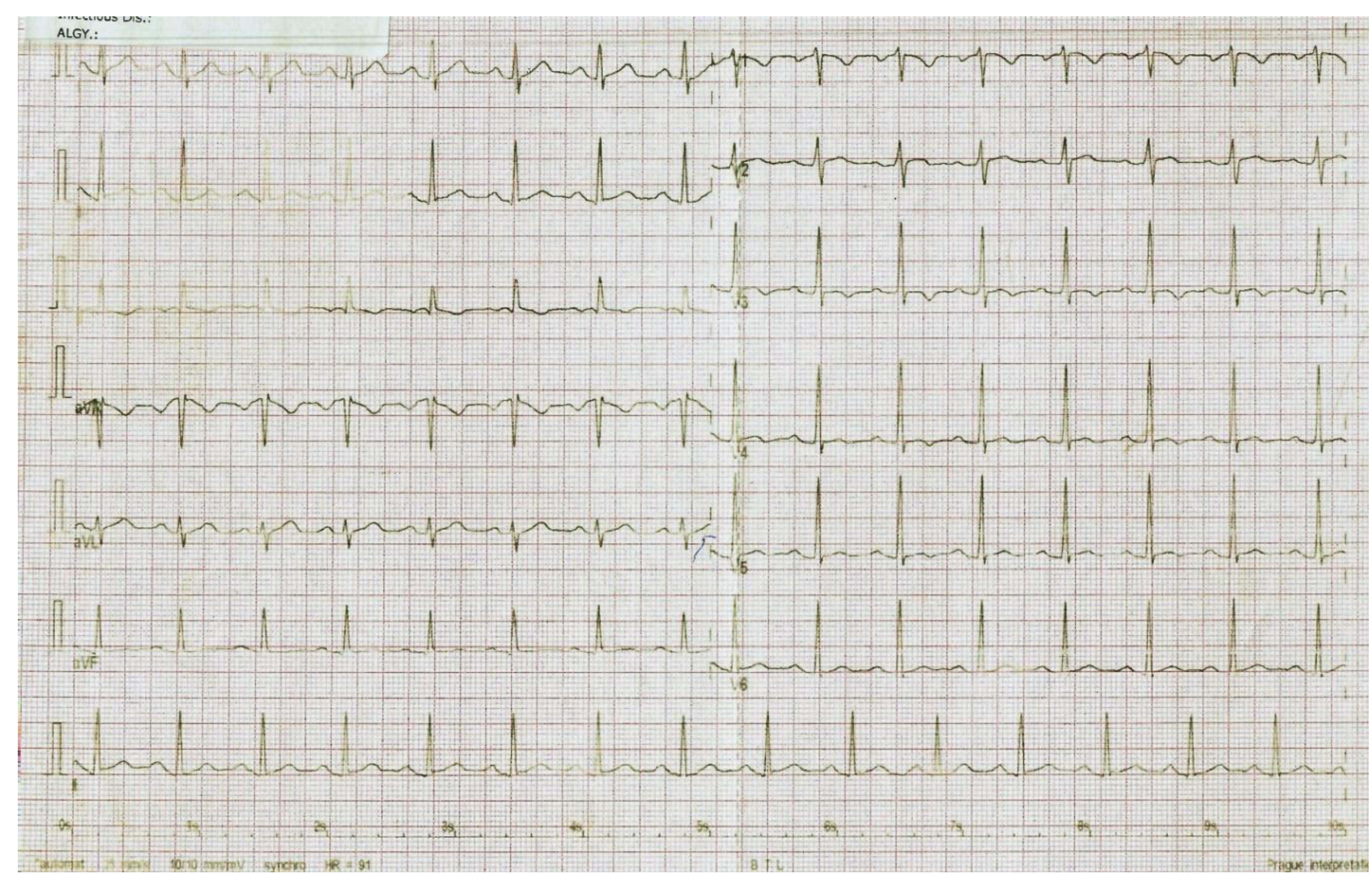

Figure 3 EKG showing normal sinus rhythm following electrical cardioversion.

sinus (NCS), which was considered the best option but had no effect on the tachycardia. A test ablation was considered on the parahisian pathway but was deemed to be associated with too high of a risk for permanent pacing, which the patient declined. The patient was discharged on medical management with the option of cryoablation for the failure of medical therapy. A 6 and 12 month's followup has been uneventful.

\section{Discussion}

To our knowledge, this is the first published case report in Sub-Saharan Africa whereby the Apple Watch was an integral part of the diagnostic process. Many patients who present to health care facilities reporting awareness of heartbeat require extensive workup: including ambulatory rhythm monitoring, noninvasive imaging, pharmacotherapy and invasive electrophysiological (EP) procedures to reach diagnosis. ${ }^{2}$ Literature from the African context shows that even though patients may present for care, most facilities are ill-equipped and lack necessary diagnostic capabilities both in human resources and equipment. ${ }^{3}$ This advancement in technology has shed light on the medical world and can therefore be considered a significant adjunct to conventional rhythm monitoring. Nevertheless, it is a dangerous practice to solely depend on it to detect heart conduction abnormalities. This technology has led to an extensive assessment of smartwatches to identify atrial fibrillation. ${ }^{4}$ The findings of this study were clinically relevant since $34 \%$ of those who received an irregular pulse notification were subsequently diagnosed to have atrial fibrillation on ECG patch readings. ${ }^{4}$ Additionally, case series have also been published whereby two patients' actual watch-based 3-lead ECG tracings corresponded to the traditional ECGs demonstrating ST-elevation myocardial infarction (STEMI), confirming the potential ability of this device also to detect myocardial ischemia. ${ }^{5}$ Only one validation study has assessed the accuracy of the wrist-worn wearable device on 51 patients who were scheduled for EPS due to a history of paroxysmal supraventricular tachyarrhythmia (SVT) or paroxysmal palpitations. This study showed an acceptable accuracy of these devices to measure baseline and induced SVT. ${ }^{6}$

\section{Conclusion}

This case highlights the importance of wearable gadgets to detect asymptomatic arrhythmia. Atrial fibrillation is 
considered most prevalent among hypertensive patients in Tanzania. ${ }^{7}$ The use of such devices will therefore aid in seeking prompt care and management. However, falsepositive notifications can trigger anxiety and increase unnecessary hospital visits. The American and European heart rhythm society has not approved this technology as a diagnostic tool ${ }^{8}$ rather a nidus for the community to seek medical advice when notified.

\section{Declaration of Patient Consent}

The authors certify that patient consent was obtained. The patient has given his consent for his images and other clinical information to be reported in the journal. The patient understands that his name and initials will not be published. The obtained consent was also forwarded to the institutional ethical committee. Institutional approval was not required to publish the case details.

\section{Funding}

There is no funding to report.

\section{Disclosure}

The authors report no conflicts of interest for this work.

\section{References}

1. Burke J, Haigney MCP, Borne R, Krantz MJ. Smartwatch detection of ventricular tachycardia: case series. HeartRhythm Case Rep. 2020;6 (10):800-804. doi:10.1016/j.hrcr.2020.08.003

2. Brugada J, Katritsis DG, Arbelo E, et al. 2019 ESC Guidelines for the management of patients with supraventricular tachycardia Task Force for the management of patients with supraventricular tachycardia of the European Society of Cardiology (ESC). Eur Heart J. 2020;41 (5):655-720.

3. Bonny A, Ngantcha M, Scholtz W, et al. Cardiac arrhythmias in Africa: epidemiology, Management Challenges, and Perspectives. $J$ Am Coll Cardiol. 2019;73(1):100-109. doi:10.1016/j.jacc.2018.09.084

4. Perez MV, Mahaffey KW, Hedlin H, et al. Large-scale assessment of a smartwatch to identify atrial fibrillation. $N$ Engl J Med. 2019;381 (20):1909-1917. doi:10.1056/NEJMoa1901183

5. Avila CO. Novel use of apple watch 4 to obtain 3-lead electrocardiogram and detect cardiac ischemia. Perm J. 2019;23.

6. Hwang J, Kim J, Choi KJ, Cho MS, Nam GB, Kim YH. Assessing accuracy of wrist-worn wearable devices in measurement of paroxysmal supraventricular tachycardia heart rate. Korean Circ J. 2019;49 (5):437-445. doi:10.4070/kcj.2018.0323

7. Bhalia S, Pallangyo P, Dalidali A, et al. Burden and correlates of atrial fibrillation among hypertensive patients attending a tertiary hospital in Tanzania. BMC Cardiovasc Disord. 2020;20(1):235. doi:10.1186/ s12872-020-01517-x

8. Slotwiner D, Varma N, Akar JG, et al. HRS expert consensus statement on remote interrogation and monitoring for cardiovascular implantable electronic devices. Heart Rhythm. 2015;12(7):e69-100.

\section{Publish your work in this journal}

The International Medical Case Reports Journal is an international, peer-reviewed open-access journal publishing original case reports from all medical specialties. Previously unpublished medical posters are also accepted relating to any area of clinical or preclinical science. Submissions should not normally exceed 2,000 words or 4 published pages including figures, diagrams and references. The manuscript management system is completely online and includes a very quick and fair peer-review system, which is all easy to use. Visit http://www.dovepress.com/testimonials.php to read real quotes from published authors. 